\title{
Electrodynamic response and stability of molecular crystals
}

\author{
Bohdan Schatschneider, ${ }^{1}$ Jian-Jie Liang, ${ }^{2}$ Anthony M. Reilly, ${ }^{3}$ Noa Marom, ${ }^{4}$ Guo-Xu Zhang, ${ }^{3}$ and Alexandre Tkatchenko ${ }^{3, *}$ \\ ${ }^{1}$ The Pennsylvania State University, Fayette, The Eberly Campus, Lemont Furnace, Pennsylvania 15456, USA \\ ${ }^{2}$ Accelrys Inc., 10188 Telesis Court, Suite 100, San Diego, California 92121, USA \\ ${ }^{3}$ Fritz-Haber-Institut der Max-Planck-Gesellschaft, Faradayweg 4-6, 14195 Berlin, Germany \\ ${ }^{4}$ Institute for Computational Engineering and Sciences, The University of Texas at Austin, Austin, Texas 78712, USA
}

(Received 30 October 2012; published 28 February 2013)

\begin{abstract}
We show that electrodynamic dipolar interactions, responsible for long-range fluctuations in matter, play a significant role in the stability of molecular crystals. Density functional theory calculations with van der Waals interactions determined from a semilocal "atom-in-a-molecule" model result in a large overestimation of the dielectric constants and sublimation enthalpies for polyacene crystals from naphthalene to pentacene, whereas an accurate treatment of nonlocal electrodynamic response leads to an agreement with the measured values for both quantities. Our findings suggest that collective response effects play a substantial role not only for optical excitations, but also for cohesive properties of noncovalently bound molecular crystals.
\end{abstract}

DOI: 10.1103/PhysRevB.87.060104

PACS number(s): 71.15.Nc, 71.45.-d, 77.22.-d

Polyacene molecular crystals form a fundamental class of aromatic solids, and have been extensively studied as potential materials for organic electronics. ${ }^{1-3}$ It is understood that the optical properties of polyacenes are very sensitive to longrange intra- and intermolecular electrodynamic interactions. This is reflected by shifts in the optical absorption frequencies upon increasing the molecule size or upon solid formation, ${ }^{4}$ and is further exhibited by the visible color of oligoacene crystals, which changes from transparent in naphthalene and anthracene, to bright orange in tetracene, and deep blue in pentacene. ${ }^{4,5}$ The optical absorption spectrum is directly related to the polarizability through the Kramers-Kronig transformation. ${ }^{6}$ Therefore, the observed changes in the optical spectrum upon crystallization of polyacenes are accompanied by a change in the molecular polarizability. In addition, these changes in polarization should directly impact the crystal lattice energy. However, the effect of electrodynamic intermolecular interactions on the cohesive properties of molecular crystals remains poorly understood. In this Rapid Communication, we show that the dipolar electrodynamic coupling between polyacene molecules reduces the solid dielectric constant by $15 \%$, and has an impact of up to $0.5 \mathrm{eV}$ per molecule on the computed van der Waals (vdW) energies and sublimation enthalpies of these molecular crystals. Our results imply that electrodynamic response is crucial for describing both the cohesive energy and the optical properties of molecular crystals, also providing strong quantitative support to empirical relations between stability and refractive index of molecular crystals. ${ }^{7}$

Polyacene crystals are extended aromatic networks characterized by polarizable $\pi$ clouds. Therefore, an appreciable part of the crystal lattice energy stems from ubiquitous attractive vdW dispersion interactions. When studying the cohesion of molecular systems, for example, using densityfunctional theory (DFT) ${ }^{8,9}$ or classical potentials, ${ }^{10}$ the vdW energy is typically computed using effective polarizabilities for hybridized "atoms" inside a molecule. It is common to approximate the frequency-dependent polarizability of every atom using a single effective excitation frequency (also called the Unsöld approximation ${ }^{11}$ ). In this model, the dipole polarizability for atom $p$ is written as

$$
\alpha_{p}(i \omega)=\frac{\alpha_{p}[n(\mathbf{r})]}{1+\left(\omega / \omega_{p}[n(\mathbf{r})]\right)^{2}},
$$

where $\alpha_{p}[n(\mathbf{r})]$ is the static polarizability of an atom $p$ and $\omega_{p}[n(\mathbf{r})]$ is the corresponding characteristic excitation frequency. In this equation we emphasize that the effective parameters can be defined as functionals of the self-consistent electron density $n(\mathbf{r})$ as done in the Tkatchenko-Scheffler (TS) method. ${ }^{12}$ Regardless of whether one treats $\alpha_{p}$ and $\omega_{p}$ as empirical parameters or obtains them from the electron density, their values for different carbon atoms in polyacene molecules and crystals turn out to be essentially degenerate (the same holds for the hydrogen atoms). This finding can be attributed to the rather similar local hybridization environment that every atom "feels" inside polyacene molecules. This simplified model for the polarizability would lead to a similar optical absorption spectrum for different polyacenes, in stark disagreement with experimental measurements and explicit excited-state calculations. ${ }^{4,5,13}$

The semilocal approximation for the polarizability in Eq. (1) neglects the dynamic electric fields that an atom experiences from all the other atoms inside a molecule or a crystal. Recently, an efficient parameter-free method was developed to include these screening effects on the polarizability for nonmetallic molecules and solids. ${ }^{14}$ We model the environment as a dipole field and solve the resulting classical Dyson-like self-consistent screening (SCS) equation, ${ }^{15-17}$

$$
\begin{aligned}
\alpha^{\mathrm{SCS}}(\mathbf{r} ; i \omega)= & \alpha^{\mathrm{TS}}(\mathbf{r} ; i \omega)+\alpha^{\mathrm{TS}}(\mathbf{r} ; i \omega) \\
& \times \int d \mathbf{r}^{\prime} \mathcal{T}\left(\mathbf{r}-\mathbf{r}^{\prime}\right) \alpha^{\mathrm{SCS}}\left(\mathbf{r}^{\prime} ; i \omega\right),
\end{aligned}
$$

where $\alpha^{\mathrm{TS}}(\mathbf{r} ; i \omega)$ is the sum of the TS effective atomic polarizabilities, ${ }^{12}$ and $\mathcal{T}\left(\mathbf{r}-\mathbf{r}^{\prime}\right)$ is the dipole-dipole interaction tensor (Hartree atomic units are used throughout). Equation (2) is discretized using atomic positions as a basis, and then solved directly and exactly by inverting the tensor corresponding to the coupled dipoles modeled as quantum harmonic oscillators $(\mathrm{QHO})$. The $\mathrm{QHO}$ parameters are defined using the TS polarizability. ${ }^{14}$ The solution of Eq. (2) yields 
the nonlocal molecular polarizability tensor $\alpha_{p q, i j}^{\mathrm{SCS}}(i \omega)$, where indices $p$ and $q$ label the atoms while indices $i$ and $j$ label the atomic Cartesian coordinates. The contraction of the molecular tensor for every atom $p$ yields the atomic polarizability tensors $\alpha_{p, i j}^{\mathrm{SCS}}(i \omega)$. These tensors now include both the short-range hybridization effects from the TS method and the long-range response screening from the solution of the SCS equation.

The electrodynamic response included upon solving the SCS equation [Eq. (2)] allows one to correctly capture two important contributions to the polarizability: (i) (de)polarization and (ii) polarizability anisotropy. The local "atom-in-amolecule" polarizability as defined in Eq. (1) leads to an essentially isotropic response for molecules and solids. ${ }^{14}$ The directionality of the polarization, well known for polyacenes from experiments ${ }^{18}$ and calculations, ${ }^{13}$ emerges from the intrinsic anisotropy of the molecular orbitals and the electrodynamic coupling between them. In the SCS formalism of Eq. (2), the anisotropy of the molecular polarizability stems from the coupling between fluctuating QHOs. For a set of small organic molecules, the SCS calculation significantly reduces the error in the molecular anisotropy to $23 \%$ from $80 \%$ in the TS method. ${ }^{14}$ The emergence of polarizability anisotropy is the main effect brought by the inclusion of electrodynamic response effects for small gas-phase molecules. In the solid state or for larger molecules, the situation is more complex. In a crystalline environment every atom experiences the electric field from the atoms within the same molecule (similar to the gas phase), as well as the field produced by neighboring molecules. While the screening of the molecule in the crystal leads to an anisotropic polarizability, one also typically finds an appreciable change in the isotropic polarizability of the molecule when compared to the gas phase.

In order to assess the relative importance of electrodynamic response on the properties of noncovalently bound molecular crystals, we have chosen to examine a series of polyacene crystals, ranging from naphthalene to pentacene. This choice allows us to study the evolution of response properties of molecular crystals and their stability as a function of molecular size and crystal environment. Initial crystal structures for each polyacene were obtained from the lowest temperature data sets available in the Cambridge Structural Database. ${ }^{19}$ The low-temperature polymorphs were chosen for tetracene and pentacene. The crystal unit cells and the internal geometries were fully optimized using DFT with the generalized gradient approximation of Perdew, Burke, and Ernzerhof (PBE) ${ }^{20}$ with vdW interactions treated using the TS method $^{12}$ (denoted as PBE+vdW). The CASTEP code was used for all calculations..$^{21,22}$ Norm-conserving pseudopotentials were employed for carbon, where valence states included the $2 s$ and $2 p$ electrons. The plane-wave basis set cutoff was set to $750 \mathrm{eV}$, ensuring that the total energy and unit cell volume were converged, as demonstrated in the study of crystalline indole and tetracyanoethylene. ${ }^{23,24}$ The $k$-point grid was kept to maintain a spacing of $0.07 \AA^{-1}$. Explicit all-electron calculations using the FHI-aims code ${ }^{25}$ confirm that the binding energies from the pseudopotential calculations are converged to better than $0.01 \mathrm{eV}$ per molecule.

The optimized $\mathrm{PBE}+\mathrm{vdW}$ lattice parameters for polyacenes along with $\mathrm{x}$-ray measurement results are shown in Table I. The overall deviations between our calculations and
TABLE I. Unit cell parameters for polyacene molecular crystals determined from LDA, PBE, and PBE+vdW calculations and lowtemperature $\mathrm{x}$-ray experiments. The dielectric constants are reported using the Clausius-Mossotti equation corresponding to Eq. (1) $\left(\varepsilon_{h}\right)$ and Eq. (2) $\left(\varepsilon_{\text {full }}\right)$. Data are reported for naphthalene, anthracene, tetracene, and pentacene.

\begin{tabular}{lllllll}
\hline \hline & $a(\AA)$ & $b(\AA)$ & $c(\AA)$ & $\rho(\mathrm{g} / \mathrm{ml})$ & $\varepsilon_{\text {full }}$ & $\varepsilon_{h}$ \\
\hline Naphthalene & & & & & & \\
LDA & 7.819 & 5.765 & 8.414 & 1.379 & & \\
PBE & 9.449 & 6.353 & 9.061 & 0.930 & & \\
PBE+vdW & 8.117 & 5.897 & 8.647 & 1.244 & 3.06 & 3.58 \\
Expt. & 8.108 & 5.940 & 8.647 & 1.239 & $3.2^{26}$ & \\
Anthracene & & & & & & \\
LDA & 8.210 & 5.773 & 10.877 & 1.428 & & \\
PBE & 9.825 & 6.350 & 11.447 & 0.984 & & \\
PBE+vdW & 8.399 & 5.906 & 11.120 & 1.313 & 3.24 & 3.80 \\
Expt. & 8.414 & 5.990 & 11.095 & 1.297 & $3.2^{4}$ & \\
Tetracene & & & & & & \\
LDA & 5.887 & 7.499 & 12.578 & 1.465 & & \\
PBE & 6.427 & 9.305 & 13.935 & 1.018 & & \\
PBE+vdW & 6.050 & 7.706 & 13.030 & 1.343 & 3.31 & 3.89 \\
Expt. & 6.056 & 7.838 & 13.010 & 1.323 & & \\
Pentacene & & & & & & \\
LDA & 6.131 & 7.427 & 13.937 & 1.502 & & \\
PBE & 6.521 & 9.361 & 15.676 & 1.032 & & \\
PBE+vdW & 6.129 & 7.676 & 14.531 & 1.392 & 3.44 & 4.08 \\
Expt. & 6.239 & 7.636 & 14.333 & 1.397 & $2.7-3.89^{18,27}$ & \\
\hline \hline
\end{tabular}

experiments are less than $2 \%$ in lattice parameters and unit cell volumes. Similar agreement is also found for the internal molecular geometries, for which the PBE+vdW method predicts the $\mathrm{C}-\mathrm{C}$ distances with an accuracy of $2 \%$ in comparison with X-ray measurements. The polyacene crystal densities predicted by the PBE+vdW method are slightly higher than the experimental ones (except for pentacene), consistent with the fact that the inclusion of zero-point energy and thermal expansion will decrease the density of the crystal. Without the inclusion of $\mathrm{vdW}$ interactions, the standard PBE functional leads to unit cell volumes that are too large by $\approx 25 \%$. In contrast, the local-density approximation (LDA) yields unit cell volumes than are $10 \%$ smaller than the experimental ones for naphthalene, anthracene, and tetracene.

To illustrate the importance of electrodynamic response in polyacene crystals, we have computed the solid dielectric constant, $\varepsilon$, using the Clausius-Mossotti formula. The required polarizabilities were obtained from (i) Eq. (1), which only includes local hybridization effects, and (ii) Eq. (2), which properly accounts for electrodynamic response screening. Comparison between the "hybridized" $\varepsilon_{h}$ and the "full" $\varepsilon_{\text {full }}$ dielectric constants in Table I reveals the importance of electrodynamic interactions. The $\varepsilon_{\text {full }}$ dielectric constants for all polyacene solids are decreased by $15 \%$ when compared with $\varepsilon_{h}$. The fully screened dielectric constants are in excellent agreement with the measured values of $\varepsilon^{4,18,26,27}$ For pentacene, $\varepsilon_{\text {full }}$ is also close to the values of 3.2 and 3.6, obtained by Sharifzadeh et al. ${ }^{3}$ from $G W$ calculations within the random phase approximation (RPA). Indeed, the SCS method solves the RPA equation for a collection of 
QHOs in the dipole approximation. This explains the good agreement with $G W$ dielectric constant and also the fact that the SCS model successfully reproduces the measured dielectric constants of crystalline silicon and germanium. ${ }^{14,28}$ We note that the calculated dielectric constants from SCS allow us to approximately determine the fundamental band gap of a molecular crystal. The fundamental gap of a molecular crystal is reduced with respect to that of a molecule in the gas phase, owing to the dielectric screening, which reduces the energy needed for adding or removing an electron. To evaluate the fundamental gap of crystalline pentacene we may use the polarization model, whereby the gap of the gas phase molecule is reduced by $2 P$. The polarization energy, $P$ (in atomic units), is given by $-(\varepsilon-1) /(2 R \varepsilon)$, where $R$ is determined from the volume per molecule in the unit cell as $R=\left[3 V_{\text {cell }} /(8 \pi)\right]^{1 / 3}$ (for two molecules per cell). ${ }^{3,29,30}$ Using cell parameters calculated with $\mathrm{PBE}+\mathrm{vdW}$ and $\varepsilon_{\text {full }}$ we obtain $P=-1.17 \mathrm{eV}$. Applying the polarization model to the gas-phase gap of $4.57 \mathrm{eV}$, obtained from a $G W$ calculation based on a consistent starting point, as described in Ref. 31, we obtain a bulk gap of $2.22 \mathrm{eV}$ in good agreement with experiment $^{32}$ and with explicit $G W$ calculations for pentacene crystal. ${ }^{3}$ We note that the optical gap is further reduced with respect to the fundamental gap due to excitonic effects, ${ }^{3,13,18}$ which are not accounted for by the SCS model.

Experimentally, the stability of molecular crystals is measured in terms of their sublimation enthalpy, i.e., the energy required to convert a certain amount of molecules from the crystalline phase to the gas phase. The sublimation process is carried out at a given temperature under constant pressure. The sublimation temperature is largely determined by the magnitude of the cohesive forces in the crystal. For polyacene crystals, the sublimation temperature varies from $\approx 300 \mathrm{~K}$ for naphtalene to $\approx 500 \mathrm{~K}$ for pentacene, ${ }^{33}$ illustrating the increase in crystal stability for larger acene molecules. There are numerous experiments that measure the sublimation enthalpies of polyacenes; in Table II we report a range of available values, extrapolated to $0 \mathrm{~K} .{ }^{33}$ We have only taken those values that are recommended as reliable after critical revision by the authors of Ref. 33, thus avoiding anomalously small or large sublimation enthalpies. Both naphthalene and anthracene crystals have been vigorously studied, and their sublimation enthalpies are well known with a spread of $0.05 \mathrm{eV}$ and $0.12 \mathrm{eV}$, respectively. There are fewer measurements available for

TABLE II. Lattice energies of polyacene crystals including zero-point energy ( $\mathrm{PBE}+\mathrm{MBDh}$ and $\mathrm{PBE}+\mathrm{MBD}$ calculations using optimized PBE+vdW geometries from Table I). The range of experimental ("Expt.") "lattice energies" from Ref. 33 extrapolated to $0 \mathrm{~K}$. Also shown are the $\Delta H$ from room temperature $(298 \mathrm{~K})$ to 0 $\mathrm{K}$ calculated from an integral over experimental $C_{p}(T)$ data, and the harmonic zero-point energy calculated using the PBE+vdW method. All values are in units of $\mathrm{eV}$.

\begin{tabular}{cccccc}
\hline \hline & $\Delta H$ & ZPE & PBE+MBDh & PBE+MBD & Expt. \\
\hline 2A & 0.041 & 0.069 & -0.993 & -0.862 & -0.803 to -0.752 \\
3A & 0.034 & 0.078 & -1.433 & -1.206 & -1.148 to -1.024 \\
4A & -0.016 & 0.110 & -1.951 & -1.587 & -1.525 to -1.299 \\
5A & -0.032 & 0.115 & -2.501 & -2.018 & -2.082 to -1.533 \\
\hline \hline
\end{tabular}

tetracene and pentacene, and for the latter the three available experimental values deviate by $0.55 \mathrm{eV}$.

In order to compare theoretical lattice energies to the measured sublimation enthalpies, the sublimation enthalpies need to be extrapolated to zero temperature by adding the enthalpy difference $\left[H_{c}^{0}(T)-H_{c}^{0}(0)\right]-\left[H_{g}^{0}(T)-H_{g}^{0}(0)\right]$, where the subscript $c$ refers to the crystal, whereas $g$ refers to the gas phase. In this work, we have calculated this enthalpy difference by integrating the measured heat capacity $C_{p}(T)$ for acene crystals, and the extrapolated gas-phase heat capacity from Refs. 34-37. Even at $0 \mathrm{~K}$, the sublimation enthalpy includes zero-point vibrational effects, and these have to be considered when comparing calculated lattice energies to the experimental enthalpy extrapolated to $0 \mathrm{~K}$. Here we determined the zero-point energy (ZPE) from phonon calculations using the $\mathrm{PBE}+\mathrm{vdW}$ method with the supercell formalism in CASTEP. ${ }^{21}$ Special care has been taken to converge the supercell size and plane-wave cutoff when performing phonon calculations. We estimate that our ZPE calculations are converged to $5 \mathrm{meV} /$ molecule. Note that vdW interactions contribute significantly to the ZPE energy and have to be included in phonon calculations to reach this level of accuracy.

Now we analyze the impact of electrodynamic response on the sublimation enthalpies of polyacene crystals. For this purpose we combine the PBE functional with the recently developed many-body dispersion (MBD) method. ${ }^{14}$ The MBD energy expression computes the long-range many-body dispersion energy to infinite order for molecules and solids with a finite band gap. In the MBD method, the full electronic system is mapped to a system of quantum harmonic oscillators. The QHO polarizabilities can be obtained either from Eq. (1) (from now on called the DFT+MBDh method) or Eq. (2) (from now on called the DFT+MBD method), thus allowing us to clearly assess the effect of electrodynamic screening on the dispersion energy and stability of molecular crystals. The ZPE-inclusive lattice energies obtained with both methods are shown in Table II and compared with experimental data.

The inspection of Table II illustrates the crucial importance of electrodynamic response for the stability of polyacene crystals. The $\mathrm{PBE}+\mathrm{MBDh}$ method uses semilocal hybridized polarizabilities and overestimates the experimental sublimation enthalpies by more than $0.20 \mathrm{eV}$ for naphthalene and up to $0.44 \mathrm{eV}$ for pentacene. Upon including the response screening, as depolarization reduces the stability of naphthalene by $0.13 \mathrm{eV}$ and of pentacene by $0.48 \mathrm{eV}$ compared to the PBE+MBDh approach. To assess the influence of the underlying DFT functional, we have also carried out calculations using the PBE-based hybrid functional, PBE0 ${ }^{38,39}$ combined with the MBD method. The PBE0 functional describes the electrostatic and inductive intermolecular interactions more accurately. ${ }^{14,40}$ However, we found that the PBE0+MBD approach yields essentially the same lattice energies as the $\mathrm{PBE}+\mathrm{MBD}$ method for all polyacenes (within $10 \mathrm{meV}$ per molecule).

The remaining slight overestimation of lattice energies in Table II compared to the experimental range can be explained by the fact that the sublimation enthalpy is measured at finite temperature, where the unit cell undergoes thermal expansion. When using the experimental unit cell at $295 \mathrm{~K}$ for naphthalene, the PBE+MBD method yields a lattice energy 

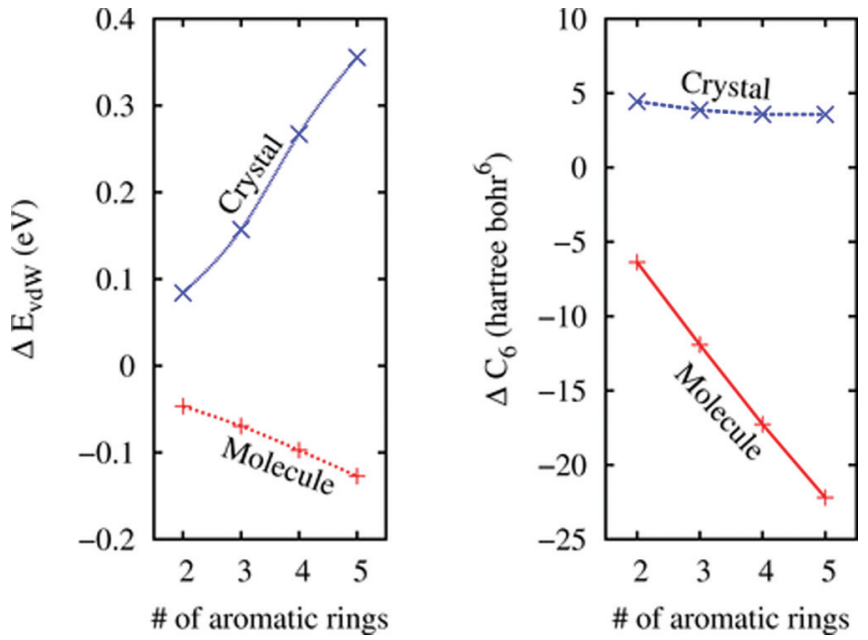

FIG. 1. (Color online) Left: Difference in the vdW energy between the PBE+MBD method using screened polarizabilities of Eq. (2) and the PBE+MBDh method based on "atom-in-a-molecule" polarizabilities of Eq. (1). Right: Difference in the $C_{6}$ coefficients between $\mathrm{PBE}+\mathrm{MBD}$ and $\mathrm{PBE}+\mathrm{MBDh}$ methods per carbon atom.

that is increased by $50 \mathrm{meV}$, essentially within the experimental range reported in Table II. We note that in contrast to PBE+MBD, the standard PBE functional predicts essentially the same lattice energy of $\approx-0.10 \mathrm{eV}$ for all polyacenes. The LDA functional does recover the trend of larger lattice energies with increasing molecule size, but underestimates the lattice energy by $0.06 \mathrm{eV}$ for naphthalene, $0.14 \mathrm{eV}$ for anthracene, and $\approx 0.20 \mathrm{eV}$ for tetracene and pentacene, compared to the highest (most positive) experimental estimates for the lattice energy.

Finally, we explain the observed difference between the $\mathrm{PBE}+\mathrm{MBDh}$ and $\mathrm{PBE}+\mathrm{MBD}$ methods by analyzing the $\mathrm{vdW}$ dispersion energies in the gas and crystal phases in Fig. 1. Along with vdW energies, we also show the change in the vdW $C_{6}$ coefficient per carbon atom upon inclusion of the electrodynamic response in the MBD method. For the gas-phase molecules, there is a significant dipole polarization along the long molecular axis, which increases the molecular polarizabilities and $C_{6}$ coefficients, leading to an increase of the vdW energy in the PBE+MBD method when compared to $\mathrm{PBE}+\mathrm{MBDh}$. However, since the $\mathrm{vdW}$ energy contribution to the molecular stability is relatively small, the change due to electrodynamic response is only $-0.05 \mathrm{eV}$ for naphthalene and up to $-0.13 \mathrm{eV}$ for pentacene. The electrodynamic response gives rise to a radically different situation in the crystal phase; in this case the interaction with neighboring molecules leads to overall depolarization, decreasing the $C_{6}$ coefficients by roughly a constant amount, when compared to the gas-phase molecules. However, the vdW energy makes a larger absolute contribution to the stability of acene crystals. This explains the sharp decrease of the vdW energy in the crystal predicted by the PBE+MBD method when compared to the PBE+MBDh approach. Overall, the opposite effect of screening for the gas-phase molecule and the crystal rationalizes the significant reduction of the lattice energy, shown in Table II, upon including electrodynamic response in the PBE+MBD method.

In summary, we have quantitatively established a connection between collective electrodynamic response and the stability of molecular crystals. Our results demonstrate that molecular crystals are significantly more complex than a simple collection of constituent molecules, and that electrodynamic response is crucial for explaining many of the unique properties of molecular crystals. We provide a quantification of the influence of electrodynamic response on sublimation enthalpies of polyacene crystals, and it is not unreasonable to expect that our findings will hold in general for other classes of molecular solids.

A.T. acknowledges support from the European Research Council (ERC Starting Grant VDW-CMAT). B.S. acknowledges support from the Eberly Science Foundation. *tkatchenko@fhi-berlin.mpg.de

${ }^{1}$ S. R. Forrest, Nature (London) 428, 911 (2004).

${ }^{2}$ T. W. Kelley, P. F. Baude, C. Gerlach, D. E. Ender, D. Muyres, M. A. Haase, D. E. Vogel, and S. D. Theiss, Chem. Mater. 16, 4413 (2004).

${ }^{3}$ S. Sharifzadeh, A. Biller, L. Kronik, and J. B. Neaton, Phys. Rev. B 85, 125307 (2012).

${ }^{4}$ M. Pope and C. E. Swenberg, Electronic Processes in Organic Crystals and Polymers (Oxford University Press, New York, 1999).

${ }_{5}^{5}$ J. E. Anthony, Ang. Chem. Int. Ed. 47, 452 (2008).

${ }^{6}$ J. S. Toll, Phys. Rev. B 104, 1760 (1956).

${ }^{7}$ K. Shimizu, M. Tariq, M. Gomes, L. Rebelo, and J. Lopes, J. Phys. Chem. B 114, 5831 (2010).

${ }^{8}$ M. A. Neumann and M. A. Perrin, J. Phys. Chem. B 109, 15531 (2005).

${ }^{9}$ D. A. Bardwell, C. S. Adjiman, Y. A. Arnautova, E. Bartashevich, S. X. M. Boerrigter, D. E. Braun, A. J. Cruz-Cabeza, G. M. Day, R. G. Della Valle, G. R. Desiraju, B. P. van Eijck, J. C. Facelli, M. B.
Ferraro, D. Grillo, M. Habgood, D. W. M. Hofmann, F. Hofmann, K. V. J. Jose, P. G. Karamertzanis, A. V. Kazantsev et al., Acta Crystallogr. Sect. B 67, 535 (2011).

${ }^{10}$ S. Wen, K. Nanda, Y. Huang, and G. Beran, Phys. Chem. Chem. Phys. 14, 7578 (2012).

${ }^{11}$ A. J. Stone, The Theory of Intermolecular Forces (Oxford University Press, New York, 1996).

${ }^{12}$ A. Tkatchenko and M. Scheffler, Phys. Rev. Lett. 102, 073005 (2009).

${ }^{13}$ M. L. Tiago, J. E. Northrup, and S. G. Louie, Phys. Rev. B 67, 115212 (2003).

${ }^{14}$ A. Tkatchenko, R. A. DiStasio, Jr., R. Car, and M. Scheffler, Phys. Rev. Lett. 108, 236402 (2012).

${ }^{15}$ B. U. Felderhof, Physica 76, 486 (1974).

${ }^{16}$ D. W. Oxtoby and W. M. Gelbart, Mol. Phys. 29, 1569 (1975).

${ }^{17}$ B. T. Thole, Chem. Phys. 59, 341 (1981).

${ }^{18}$ D. Faltermeier, B. Gompf, M. Dressel, A. K. Tripathi, and J. Pflaum, Phys. Rev. B 74, 125416 (2006). 
${ }^{19}$ F. H. Allen, Acta Crystallogr. Sect. B 58, 380 (2002).

${ }^{20}$ J. P. Perdew, K. Burke, and M. Ernzerhof, Phys. Rev. Lett. 77, 3865 (1996).

${ }^{21}$ S. J. Clark, M. D. Segall, C. J. Pickard, P. J. Hasnip, M. J. Probert, K. Refson, and M. C. Payne, Z. Kristallogr. 220, 567 (2005).

${ }^{22}$ E. R. McNellis, J. Meyer, and K. Reuter, Phys. Rev. B 80, 205414 (2009).

${ }^{23}$ B. Schatschneider and J.-J. Liang, J. Chem. Phys. 135, 164508 (2011).

${ }^{24}$ B. Schatschneider, J.-J. Liang, S. Jezowksi, and A. Tkatchenko, CrystEngComm 14, 4656 (2012).

${ }^{25}$ V. Blum, R. Gehrke, F. Hanke, P. Havu, V. Havu, X. Ren, K. Reuter, and M. Scheffler, Comput. Phys. Commun. 180, 2175 (2009).

${ }^{26}$ T. Suthana, N. Rajesha, P. Dhanaraja, and C. Mahadevan, Spectrochim. Acta Part A 75, 69 (2010).

${ }^{27}$ C. H. Kim, O. Yaghmazadeh, D. Tondelier, Y. B. Jeong, Y. Bonnassieux, and G. Horowitz, J. Appl. Phys. 109, 083710 (2011).

${ }^{28}$ V. V. Gobre, R. A. DiStasio Jr., R. Car, M. Scheffler, and A. Tkatchenko (unpublished).

${ }^{29}$ I. G. Hill, A. Kahn, Z. G. Soos, and R. A. Pascal, Jr., Chem. Phys. Lett. 327, 181 (2000).
${ }^{30}$ J. B. Neaton, M. S. Hybertsen, and S. G. Louie, Phys. Rev. Lett. 97, 216405 (2006).

${ }^{31}$ T. Körzdörfer and N. Marom, Phys. Rev. B 86, 041110 (2012).

${ }^{32}$ E. A. Silinsh, V. A. Kolesnikov, I. J. Muzikante, and D. R. Balode, Phys. Status Solidi B 113, 379 (1982).

${ }^{33}$ M. V. Roux, M. Temprado, J. S. Chickos, and Y. Nagano, J. Phys. Chem. Ref. Data 37, 1855 (2008).

${ }^{34}$ NIST WebBook and references within, http://webbook.nist.gov/ chemistry/name-ser.html

${ }^{35}$ J. P. McCullough, H. L. Finke, J. F. Messerly, T. C. Kincheloe, and G. Waddington, J. Phys. Chem. 61, 1105 (1957).

${ }^{36}$ P. Goursot, H. L. Girdhar, and E. F. Westrum, Jr., J. Phys. Chem. 74, 2538 (1970).

${ }^{37}$ W.-K. Wong and E. F. Westrum, Jr., Mol. Cryst. Liq. Cryst. 61, 207 (1980).

${ }^{38}$ J. P. Perdew, M. Ernzerhof, and K. Burke, J. Chem. Phys. 105, 9982 (1996).

${ }^{39}$ C. Adamo and V. Barone, J. Chem. Phys. 110, 6158 (1999).

${ }^{40}$ B. Santra, J. Klimeš, D. Alfè, A. Tkatchenko, B. Slater, A Michaelides, R. Car, and M. Scheffler, Phys. Rev. Lett. 107, 185701 (2011). 